\title{
Fine Mapping Suggests that the Goat Polled Intersex Syndrome and the Human Blepharophimosis Ptosis Epicanthus Syndrome Map to a 100-kb Homologous Region
}

\author{
Laurent Schibler, Edmond P. Cribiu, Anne Oustry-Vaiman, Jean-Pierre Furet, \\ and Daniel Vaiman ${ }^{1}$ \\ Institut National de la Recherche Agronomique (INRA), Département de Génétique Animale, Laboratoire de Génétique \\ biochimique et de Cytogénétique, 78350 Jouy-en-Josas, France
}

\begin{abstract}
To clone the goat Polled Intersex Syndrome (PIS) gene(s), a chromosome walk was performed from six entry points at 1q43. This enabled 91 BACs to be recovered from a recently constructed goat BAC library. Six BAC contigs of goat chromosome 1q43 (ICCl-ICC6) were thus constructed covering altogether $4.5 \mathrm{Mb}$. A total of 37 microsatellite sequences were isolated from this $4.5-\mathrm{Mb}$ region (16 in this study), of which 33 were genotyped and mapped. ICC3 $(1500 \mathrm{~kb})$ was shown by genetic analysis to encompass the PIS locus in a $\sim 400-\mathrm{kb}$ interval without recombinants detected in the resource families (293 informative meioses). A strong linkage disequilibrium was detected among unrelated animals with the two central markers of the region, suggesting a probable location for PIS in $\sim 100 \mathrm{~kb}$. High-resolution comparative mapping with human data shows that this DNA segment is the homolog of the human region associated with Blepharophimosis Ptosis Epicanthus inversus Syndrome (BPES) gene located in 3q23. This finding suggests that homologous gene(s) could be responsible for the pathologies observed in humans and goats.
\end{abstract}

[The sequence data, PCR primers and PCR conditions for STS and microsatellites described in this paper have been submitted to the GenBank data library under accession nos. AQ666547-AQ666579, AQ686084-AQ686129, AQ793920-793931, AQ810429-AQ810527, G41201-G41228, and G54270-G54286.]

In mammals, sex determination mechanisms act with an exquisite precision to avoid the occurrence of intersex phenotypes. The bandmaster of the sex-determination pathway is the SRY gene, located on the Y chromosome, whose action is sufficient to induce maleness in XX mice (Koopman et al. 1991). The behavior of the downstream actors, whose roles are to amplify the primary decision, is much less documented for two main reasons. The first reason is the complexity of the pathway (Swain and Lovell-Badge 1999); sex determination occurs relatively late in embryonic development, which implies the existence of complex coordination mechanisms between one cell lineage and another. The second reason is that an unknown number of genes remain to be identified inside the sex determination cascade. The cloning of these genes is the prerequisite to understanding mammalian sex determination as a whole. One approach for breaking down sex determination into its components is the analysis of genetic cases of sex reversal. In humans, the scarcity of familial cases impedes classical linkage analysis. Despite this difficulty, success has been achieved in a few

\footnotetext{
'Corresponding author.
}

E-MAIL vaiman@biotec.jouy.inra.fr; FAX 0033134652478. cases, such as SOX9 (Tommerup et al. 1993; Foster et al. 1994), implicated in XY sex reversal, SOX3 (Stevanovic et al. 1993; Collignon et al. 1996) and DAX1 (Zanaria et al. 1995), both putatively playing a role in ovarian differentiation. In 1996, we located the first mammalian gene involved in XX sex reversal, the goat PIS (Polled Intersex Syndrome) locus (Vaiman et al. 1996a). One peculiarity of this syndrome is the full association between absence of horns and intersexuality (Asdell 1944). No recombinant has ever been observed between the two phenotypes (Ricordeau and Lauvergne 1967; Soller et al. 1969), suggesting that they are either under the control of a single pleiotropic gene or two very closely linked genes. Whereas intersexuality appears as autosomal recessive, polledness is autosomal dominant, which has been helpful in building resource families (Vaiman et al. 1996a). The locus was initially localized to the distal region of goat chromosome 1 . Its genetic localization was then refined by the targeted isolation and genotyping of new microsatellite markers using comparative mapping (Schibler et al. 1998a) and selected chromosomal fractions (Vaiman et al. 1999b). The PIS interval was thus reduced to a $\sim 1-\mathrm{cM}$ region of goat chromosome 1q43. The homologous human region was identified precisely as a human chromosome 
3q23 DNA segment encompassing the COP gene, at a location homologous to human Blepharophimosis Ptosis Epicanthus Syndrome (BPES) (Zlotogora et al. 1983; Deng et al. 1991; Toomes and Dixon 1998). In this study, we describe the construction of six contig elements from the goat region (a total of $4.5 \mathrm{Mb}$ ), locate PIS in one of these contigs, and refine the genetic localization by linkage disequilibrium mapping to $0.1 \mathrm{cM}(\sim 100 \mathrm{~kb}$, according to the contig data). We suggest that goat PIS and human BPES could be encoded by a homologous gene.

\section{RESULTS}

\section{Comparative Mapping with Human ESTs}

A total of 85 primer pairs corresponding to 60 ESTs (mainly from 3'-untranslated regions) mapped on the human gene map between TFDP 2 and NCK were tested for amplification on goat genomic DNA. Only 22 ESTs (36\%) were found to amplify goat DNA with sufficient specificity and were used to screen the goat BAC library. FISH mapping of these BACs led to inconsistent results in 16 cases. Only BACs corresponding to six human ESTs WI-13749, WI-9573, STSG3182, WI-7255, STSG9571, and WI14619 were mapped by FISH on goat chromosome 1q43. They were thus used as starting points for bidirectional walking.

\section{Chromosome Walking and Contig Assembly}

Initial screening of the goat BAC library with human coding sequences (six ESTs from this study and two other coding sequences) resulted in eight primary contigs that were then merged into six (called ICC1-ICC6). We used a chromosome bidirectional walking strategy starting simultaneously from these multiple entry points: A total $113 \mathrm{BAC}$ end sequences were obtained for most of the clones (59 clones) and were used to sequentially isolate new BACs. Seventy STS were also derived from 23 BACs to confirm and specify overlaps. Only one end sequence could be obtained for five BAC clones. Eighteen other end sequences were found to contain large repeated regions (SINE or LINE) and thus could not be used to screen the library. Likewise, about one-third (22) of STS sequences generated were homologous to SINEs. In total, 130 sequences (92 of which are from BAC ends) were used for contig construction.

No overlapping clone could be obtained with T6 ends of 20A4 or 106F8/A7 (see ICC2 on Fig. 1). To fill in this gap, a sheep BAC library (Vaiman et al. 1999a) was screened, making it possible to isolate Oar7C4 and Oar53E5. However, further work in this contig (ICC2) was abandoned as new genetic data show that the PIS-relevant region was in ICC3.

To confirm overlap and evaluate the genomic coverage of the contigs, restriction analysis was performed using NotI and SalI. BAC insert sizes ranged from 100 to $200 \mathrm{~kb}$, with $153 \mathrm{~kb}$ on average, in good agreement with our previous estimate (Schibler et al. 1998b). Contig sizes could be estimated at 400, 1000, 1500, 1000, 400, and $300 \mathrm{~kb}$ for ICC1-ICC6, respectively. ICC2, ICC3, and ICC4 contigs (67 clones) and resulting restriction maps are shown in Figure 1. Combining all end sequences, STSs, and microsatellites, our BAC/STS map reaches an average resolution of one STS per 30 $\mathrm{kb}$. In these $4.5 \mathrm{Mb}, 3.2$ genomic coverage was observed, consistent with our previous estimation with the whole BAC library (Schibler et al. 1998b). However, a further examination of the contigs suggests that some regions are under-represented, with small overlaps between clones. Furthermore, statistical analysis reveals that some other regions displaying over seven redundancy (between and within ICC3 and ICC4), are over-represented $(P<0.005)$. As a whole, these observations suggest a preferential cloning of some regions.

\section{Linkage Analysis Determined a 400-kb Interval Without Recombinants}

During this work, a total of 35 BACs from the contigs were subcloned, and 37 microsatellites were isolated, 16 of which are reported here (Table 1 ). On average, 1.5 (TG)n microsatellite was identified in each BAC. None was found in three BACs (141F1, 296F5, and 284E7). The polymorphism of new microsatellites isolated from the ongoing contig was characterized by genotyping primarily the 12 family founder males. Genetic localization of the markers was obtained by analyzing the segregation either in all the informative families or only inside the families in which recombinants were left. Combining linkage data with BAC positions inside the contig, the marker order was established between the two anchor genes Sox14 and CRBP1 as LSCV001(LSCV116; LSCV103; LSCV056)-(LSCV120; LSCV119)-LSCV200-(LSCV204; LSCV205)(LSCV 203; LSCV202)-LSCV099-(LSCV098; LSCV095)-LSCV082-LSCV045-LSCV0 75 LSCV076-LSCV077-LSCV066. Markers in parentheses belong to the same BAC clone. Among the 293 informative meioses available, one recombinant was observed on one side between PIS and the markers LSCV120/119 (individual 501 in family 163). On the other side, one recombinant was present between PIS and LSCV045 (individual 368 in family 226). The recombinant 501 is still present for LSCV200, LSCV204, and LSCV205 but disappear at LSCV203. Symmetrically, the recombinant 368 disappear at LSCV082, meaning that the closest flanking crossovers are located in the LSCV045/LSCV082 interval and in the LSCV205/LSCV203 interval. The physical size of this interval without recombinants could be estimated at $\sim 400 \mathrm{~kb}$ by analyzing the restriction site positions in the ICC3 BAC contig. 


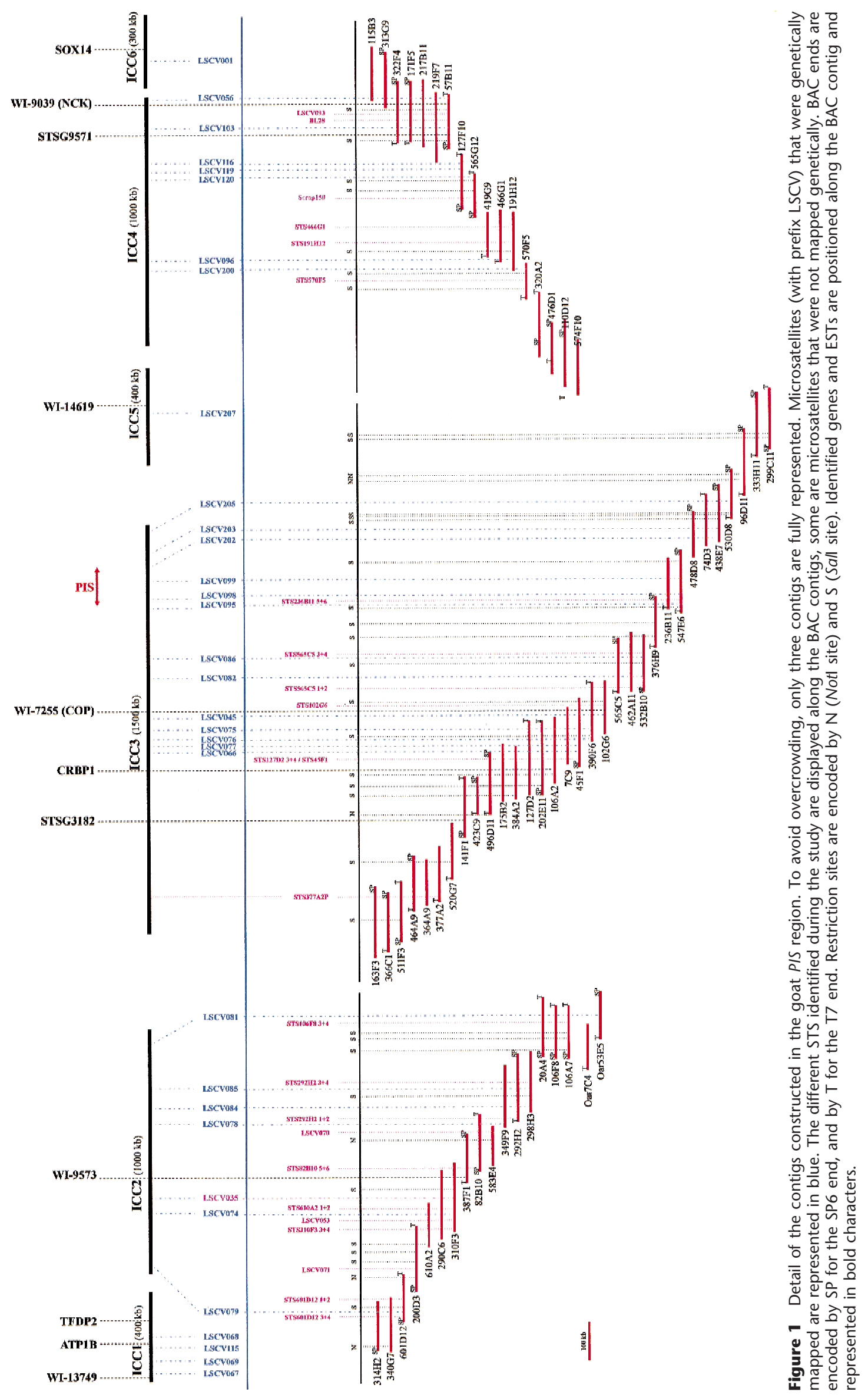


Schibler et al.

Table 1. Characteristics of New Goat Microsatellites in the PIS Region

\begin{tabular}{|c|c|c|c|c|c|c|}
\hline $\begin{array}{l}\text { Lab } \\
\text { name }\end{array}$ & $\begin{array}{c}\text { Accession } \\
\text { no. }\end{array}$ & $\begin{array}{c}\text { Repeat structure } \\
\text { of cloned } \\
\text { microsatellite }\end{array}$ & Primer 1 & Primer 2 & $\begin{array}{c}\text { Heterozy- } \\
\text { gosity }\end{array}$ & Contig \\
\hline LSCV08 & G54276 & (CA) 17 & GGGTTGCAAAGAGTCAAATAC & GAAACCACTCACACATAAGTG & 0.82 & $\operatorname{ICC} 5$ \\
\hline LSCV082 & $2 \quad 954277$ & $(\mathrm{GT}) 22$ & CAGAAACATGACTTAGAACGT & CTGTCCCTTCCAGACCCA & 0.82 & $\mathrm{ICC} 3$ \\
\hline ISCV084 & $34 \quad G 63873$ & (AC) 10 & CTGGTGGACTATAGTTCATG & CAGTCACTCTCACTTGAAAC & 0.46 & ICC5 \\
\hline LSCV085 & 35 G63868 & (CA) 14 & CTTCTGGACTGGAACTTCC & CTGTGCTGTATTAGGTTCAC & 0.55 & ICC5 \\
\hline LSCV093 & $3 \quad \mathrm{G} 63869$ & $(\mathrm{TG}) 17 \mathrm{C}(\mathrm{TG}) 8$ & GAGATTTTGCTTTCTAAAATGC & CTAATGGAAGTATTACTTTAATATCTT & n.d. & ICC2 \\
\hline LSCV095 & $5 \quad$ G54278 & $(\mathrm{AC}) 23$ & CTAATATCAAATACTCACCTGA & GCACAGCTGTGAAGTGCTA & 1.00 & $\mathrm{ICC} 3$ \\
\hline LSCV096 & $6 \quad 654279$ & (GT)6C(GT)5 & CAGTCAAGACATGGAAGCTC & CAACCACATTGCTGTGAGAT & n.d. & $\mathrm{ICC} 2$ \\
\hline LSCV098 & 8 G54281 & $(\mathrm{TG}) 4 \mathrm{C}(\mathrm{GT}) 12$ & GCCAATTCAGCCCCAATTGAG & GACTTAATGCATACGTGCACTC & 0.92 & $\mathrm{ICC} 3$ \\
\hline LSCV099 & $9 \mathrm{G} 54280$ & (TG)10TT(TG)6 & GCACTGTTGCAGGTCGTTTC & AAGCCAGGGATGCCCAAC & 0.67 & $\mathrm{ICC} 3$ \\
\hline LSCV200 & $10 \quad G 54282$ & (TG) 12 & GGACTTATCAACACATTGGAC & GTTTCTGGTACAGAGCAAGTG & 0.50 & $\mathrm{ICC} 2$ \\
\hline LSCV202 & $2 \mathrm{G} 63871$ & $(\mathrm{GT}) 22$ & CTAGTGATGCTAGATTGCCC & GGGACACCCAAGTAACTCAC & 0.73 & $\mathrm{ICC} 3$ \\
\hline LSCV203 & $3 \quad \mathrm{G} 63872$ & $(\mathrm{GT}) 14$ & ATCTTTTTGTGGAATCTACTG & TGTTAAACGAAACACTATGG & 0.64 & $\mathrm{ICC} 3$ \\
\hline LSCV204 & $4 \quad G 54284$ & (GT) 18 & ATCAGTAGTAGGAAGCCAGT & TTAAGTTATTCAGCCATTTTG & 0.33 & $\mathrm{ICC} 3$ \\
\hline LSCV205 & $5 \quad G 54283$ & $(\mathrm{TG}) 18$ & GGCACATACTGGGACCAAC & GACCCAAAGAAGTCACGAAC & 0.64 & $1 \mathrm{CC} 3$ \\
\hline LSCV206 & 6 G54286 & $(\mathrm{GT}) 14$ & GGCATTAATATCCCATCCCTG & GTTTTCTTCACCTCTTCCCTG & n.d. & $\operatorname{ICC} 5$ \\
\hline LSCV207 & 17 G54285 & (GT) 17 & GAAGCAGGGCAAGACACC & GTTATTCAGGGGTCCTGTG & 0.50 & $\mathrm{ICC} 5$ \\
\hline
\end{tabular}

\section{Linkage Disequilibrium Analysis}

Inside the non-recombinant interval, genotype analysis of the marker LSCV098 revealed a very strong linkage disequilibrium (LD) and a tight association of the polled allele with a rare allele of the microsatellite (allele 1, Fig. 2). Linkage disequilibrium was then systematically explored for all the unrelated animals available in the resource families (Table 3 , below). The goat resource families contain 246 unrelated animals, of which 12 are heterozygous males (founders), 211 are homozygous (horned) females, and 23 are heterozy- gous females. Sixty independent additional animals crossed to produce intersex progeny were also genotyped. Allele distributions were plotted in histograms for the markers in the LSCV082/LSCV203 interval (Fig. 1). $\chi^{2}$ analyses revealed significant distortions with one particular allele for LSCV098 (allele 1, 26 of 34 occurrences), LSCV095 (allele 5, 26 of 34 occurrences), and LSCV202 (allele 10, 15 of 34 occurrences). For LSCV082, distortions were visible for three alleles (5, 13 , and 14), but the $\chi^{2}$ test was not significant. For LSCV099, no significant discrepancy could be observed, probably due to the very high frequency of one allele (allele 4: $f=0.95)$. The polled animals are heterozygous for the polled phenotype (Pp), however, as these animals are male or female genitors, microsatellite alleles for carrier and non-carrier chromosomes could be easily deduced by analyzing their progeny (Table 2). Linkage disequilibrium was tested using the DISLAMB program (Terwilliger 1995). Highly significant values were obtained for LSCV095, LSCV098, and LSCV202. Conversely, LSCV082 and LSCV203 did not display significant linkage disequilibrium. Estimations of the $\lambda$ parameter were of 0.69 , 0.74, and 0.46 for LSCV095, 098 and 202, respectively. Multipoint analysis of linkage disequilibrium was carried out using the DISMULT program (Terwilliger 1995), and the resulting curve 
Table 2. Microsatellite Haplotypes in the PIS Region for Hornless Individuals

\begin{tabular}{|c|c|c|c|c|c|}
\hline & LSCV082 & PIS & LSCV095 & LSCV098 & LSCV202 \\
\hline 191 & 5 & $P$ & 4 & 1 & 10 \\
\hline 226 & 5 & $P$ & 4 & 1 & 10 \\
\hline 228 & 5 & $P$ & 4 & 1 & 10 \\
\hline 232 & 5 & $P$ & 4 & 1 & 10 \\
\hline 625 & 5 & $P$ & 4 & 1 & 10 \\
\hline 212 & 13 & $P$ & 4 & 1 & 10 \\
\hline 842 & 13 & $P$ & 4 & 1 & 10 \\
\hline 843 & 13 & $P$ & 4 & 1 & 10 \\
\hline 231 & 13 & $\mathrm{P}$ & 4 & 1 & 10 \\
\hline 229 & 13 & $\mathrm{P}$ & 4 & 1 & 10 \\
\hline 12 & 13 & $\mathrm{P}$ & 4 & 1 & 10 \\
\hline 622 & 14 & $P$ & N.D. & 1 & 10 \\
\hline 143 & 14 & $P$ & 4 & 1 & 10 \\
\hline 618 & 5 & $P$ & 4 & 1 & $x$ \\
\hline 239 & 5 & $P$ & 4 & 1 & $x$ \\
\hline 163 & 14 & $\mathrm{P}$ & 4 & 1 & $x$ \\
\hline 176 & 14 & $P$ & 4 & 1 & $x$ \\
\hline 620 & 14 & $P$ & 4 & 1 & $x$ \\
\hline 107 & 14 & $\mathrm{P}$ & 4 & 1 & $x$ \\
\hline 144 & 14 & $P$ & 4 & 1 & $x$ \\
\hline 23 & 14 & $P$ & 4 & 1 & $x$ \\
\hline 634 & 14 & $P$ & 4 & 1 & $x$ \\
\hline 230 & 14 & $P$ & 4 & 1 & $x$ \\
\hline 237 & 5 & $P$ & 4 & $x$ & $x$ \\
\hline 657 & N.D. & $P$ & 4 & $x$ & $x$ \\
\hline 329 & 5 & $P$ & $x$ & $x$ & $x$ \\
\hline 118 & 13 & $p$ & $x$ & $x$ & $x$ \\
\hline 330 & 13 & P & $x$ & $x$ & $x$ \\
\hline 402 & 13 & $P$ & $x$ & $x$ & $x$ \\
\hline 119 & 14 & $P$ & $x$ & $x$ & $x$ \\
\hline 621 & 14 & $P$ & $x$ & 1 & $x$ \\
\hline 327 & $x$ & $P$ & N.D. & 1 & $x$ \\
\hline 429 & $x$ & $P$ & N.D. & $x$ & $x$ \\
\hline 427 & 14 & $P$ & $x$ & 1 & 10 \\
\hline 403 & 14 & $P$ & $x$ & N.D. & 10 \\
\hline
\end{tabular}

cluded because of the very high frequency of its allele 4 (0.95), making it uninformative for haplotype analysis. Three haplotypes were found unmodified in 13 chromosomes (Table 3). Recombination points were observed at different positions in the other haplotypes (Table 3), which made it possible to situate the PIS between LSCV082 and LSCV095. The marker order was ascertained by PCR inside the contig. The relative position of LSCV095 and LSCV098 was ascertained by PCR on 60 subclones from a Sau3AI 10-kb sublibrary constructed by partial digestion of the $376 \mathrm{H} 9 \mathrm{BAC}$. Assuming a total distance of $300 \mathrm{~kb}$ between LSCV082 and LSCV202, the intermarker distance was estimated by measuring the proportion of recombinant chromosomes between marker pairs (from Table 3) times 300. It could therefore be estimated at $158,17,97$, and 50 $\mathrm{kb}$, for LSCV082-LSCV095, LSCV095-LSCV098, LSCV098-LSCV202, and LSCV202-LSCV203, respectively. Southern blot experiments revealed that LSCV098 and LSCV095 belonged to a common $~ 20-\mathrm{kb}$ SmaI fragment (not shown). Whereas the association is apparent with a unique allele for LSCV095 and LSCV098, three alleles of LSCV082 appeared to be frequently associated with $\mathrm{P}$ chromosomes. Although alleles 13 and 14 may derive from each other, the different size of allele 5 suggests the early occurrence of a recombination event between PIS and LSCV082. Haplotype analysis suggests that the mutation maps to the left half of the interval defined by linkage disequilibrium. As the three lod score units of the LD curve roughly corresponds to $100 \mathrm{~kb}$, the causal mutation should lie in the $50 \mathrm{~kb}$ near LSCV095, in the LSCV082LSCV095 interval. plotted (Fig. 3). The distance between LSCV082 and LSCV203 was estimated at $300 \mathrm{~kb}$, calculated on the basis of the different NotI and SalI observed restriction sites. The DISMULT curve had a steep slope with lod scores peaking at 28.5 near markers LSCV095 and LSCV098. A rough indication for positioning the mutation is given by the three lod score unit interval around this 28.5 and corresponds to a physical distance of $100-150 \mathrm{~kb}$.

\section{Haplotype Analysis in Nonrelated P Carriers}

Haplotype analysis was carried out for the heterozygote carriers of the PIS condition (a total of 35 unrelated individuals). Three haplotypes were found in PIS chromosomes for markers LSCV082, LSCV095, LSCV098, and LSCV202: 8-4-1-10, 13-4-1-10, and 144-1-10. Marker LSCV099 was not in-

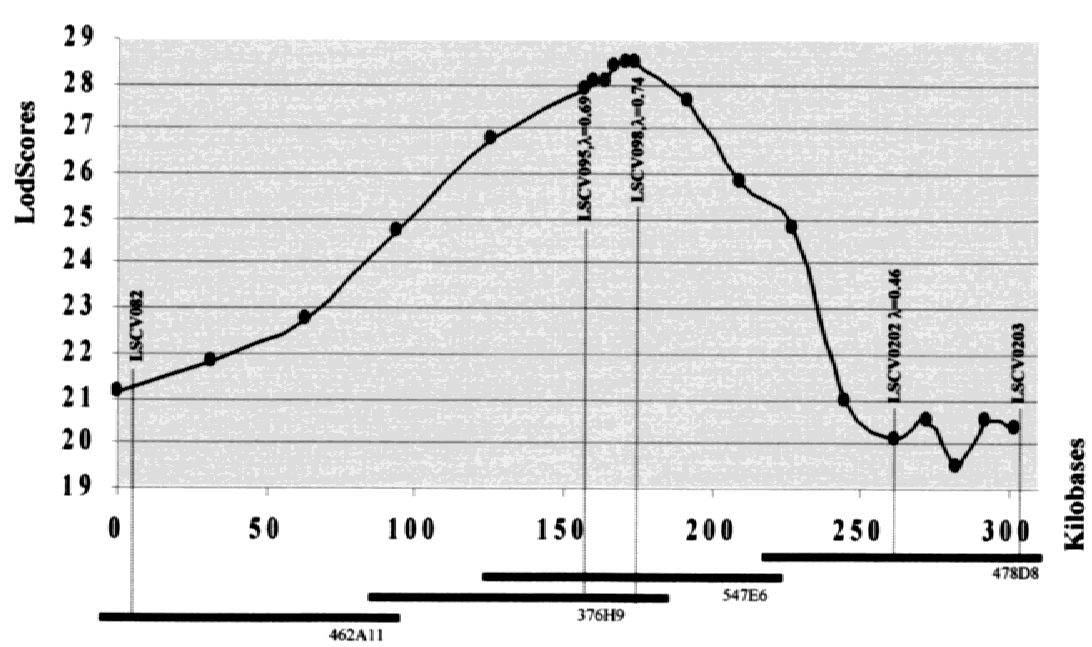

Figure 3 LD curves in the PIS region between markers LSCV203 and LSCV082. The curve displays a steep slope (eight lod score units) at markers LSCV095 and LSCV098. Values of $\lambda$ were determined by the DISLAMB program (see text) and indicated for LSCV095, LSCV098, and LSCV202, for which significant LD was found. The four BACs of the ICC 3 contig containing the five markers cover a physical distance of $\sim 300 \mathrm{~kb}$ (according to the restriction mapping profile). 


\begin{tabular}{|c|c|c|c|}
\hline Family & $\begin{array}{c}\text { Pp } \\
\text { (polled) }\end{array}$ & $\begin{array}{c}p p \\
\text { (normal) }\end{array}$ & Total \\
\hline 12 & 4 & 41 & 45 \\
\hline 23 & 1 & 16 & 17 \\
\hline 107 & 3 & 10 & 13 \\
\hline 118 & 2 & 10 & 12 \\
\hline 163 & 1 & 11 & 12 \\
\hline 176 & 1 & 9 & 10 \\
\hline 191 & 2 & 25 & 27 \\
\hline 212 & 3 & 43 & 46 \\
\hline 226 & 11 & 26 & 28 \\
\hline 327 & 4 & 10 & 14 \\
\hline 402 & 3 & 10 & 13 \\
\hline
\end{tabular}

\section{DISCUSSION}

\section{Contig Assembly Through Chromosome Walking}

Contig building in humans starts typically with scaffold YAC contigs obtained from human physical maps (Chumakov et al. 1995; Hudson et al. 1995). As BACs or PACs are more suited to fine mapping and sequencing, YAC contigs have to be translated into BAC or PAC contigs. As there are numerous STSs, microsatellites, and ESTs in humans, large BAC contigs are easily generated in this species, leaving only a few small gaps. These gaps have to be filled in later on by bidirectional walking. In livestock species however, as no overall physical map is available, contig assembly relies only on a walking strategy, starting from only two distant entry points. This can be a very tedious and timeconsuming task. Comparative mapping, exploiting the wealth of data available in humans, could be an efficient way to find new entry points and thus speed up contig construction. With genes as interspecific markers, it has been shown in a previous work that such a strategy could be effective (Schibler et al. 1998a; Vaiman et al. 1999b), leading to four starting points in the 4 -cM interval between TFDP2 and SOX14. However, closing gaps between these contig drafts requires a higher level of resolution, which could only be obtained with human ESTs. In previous work (Schibler et al. 1998a), 202 goat BACs identified with interspecific primers were mapped to the goat genome. Among BACs mapped at expected locations (according to previous comparative mapping data), a sample of 28 goat amplification products were sequenced and displayed, on average, a $90.3 \%$ similarity with the original sequence. In contrast, 10 inconsistently mapped clones were sequenced, which corresponds either to gene families or to random DNA fragments. In the same study, ESTs were much less efficient for recovering BACs than exonic sequences (23\% success), certainly because human ESTs are mainly 3' sequences, poorly conserved between ruminants and humans. In this study, the use of ESTs was even less efficient (probably owing to sampling): only 6 of 60 ESTs tested were cytogenetically mapped in the region of interest, leading to 4 new entry points. Progress in the contig construction was achieved by bidirectional walking, using BAC end sequences. This strategy, although efficient, is sequential and therefore time consuming. About 8 months were required for this contig assembly. Repetitive sequences (SINEs or LINEs), frequently encountered in either BAC ends or STS, made it more difficult by bringing the walking process abruptly to an end. This illustrates the specific problems of building contigs from low or middle redundancy libraries. In contrast, libraries of $>10$ genome equivalents obviously mitigate this problem in human physical mapping efforts. Nevertheless, our work shows that contigs larger than $1 \mathrm{Mb}$ can be constructed with threefold genomic coverage libraries.

\section{PIS Linkage Disequilibrium Mapping}

Whereas LD has been extensively used in humans or mice, positional cloning experiments in domestic animals have seldom used this approach (Dunner et al. 1997; Riquet et al. 1999). This is rather paradoxical, as the population structure in domestic ruminants seems particularly adapted to LD analysis. Artificial insemination is extensively used in dairy cattle or alpine and saanen goats, and uses a limited number of animals as progenitors. This situation is highly favorable for observing founder effects, as is the situation of the human Finnish population (de la Chapelle and Wright 1998). The factor limiting the use of LD analysis has probably been the reduced number of polymorphic markers available in these species. This limitation has been overcome in our work by the oriented production of numerous microsatellites in a limited chromosome region.

The scientific history of the PIS mutation probably starts at the end of the nineteenth century, with the description of he-goat hermaphrodites (Petit 1894; Bourdelle 1903), and the total association between this phenotype and polledness (Asdell 1944). Since 1920, hornless bucks were extensively used for reproduction throughout the world to create hornless herds. One can estimate that 100 generations followed one another from the start of human selection. Assuming a recombination rate of $\theta$, the number of observed nonrecombinant chromosomes between gene and marker can be roughly computed as $\mathrm{NR}=(1-\theta)^{\mathrm{n}}$. With LSCV095 or LSCV098, for which 8 recombinants of 35 (Table 2) are observed in carrier chromosomes, this corresponds to a distance of $0.26 \mathrm{cM}$. The three lod score unit interval suggests even a smaller region of $0.1 \mathrm{cM}$ (Fig. 3). Finally, haplotype analysis suggests that the mutation is located between LSCV095 and LSCV082, which are separated by a physical distance of $\sim 150 \mathrm{~kb}$.

The linkage disequilibrium demonstrated here suggests that all of the animals used in this study, al- 
though belonging to two different goat breeds, saanen and alpine, can probably be traced back to a single common ancestor (as LSCV098 allele 1 is rare in the population and closely associated to the PIS state). The shape of the LD curve (output from DISMULT) is almost symmetrical, giving no indication of any recombination hot-spot in the region. There is clearly a steep increase in lod score from 21.5 to 28.5 near the two very close markers LSCV095 and LSCV098, demonstrating that the LD affects only a short region. Interestingly, whereas the haplotype analysis map the mutation between LSCV095 and LSCV082, the highest LD is observed on the other side for LSCV098. These apparently conflicting results are probably due to the low frequency of LSCV098 allele $1(<0.1)$, associated with the mutation. In contrast, allele 4 of LSCV095 is much more frequent $(0.23)$, rendering the disequilibrium less conspicuous. In any case, the $\lambda$ values are very similar for these two markers and probably indicate that the causal mutation has to be searched in the vicinity of LSCV095. The sharp peak of the LD curve is rarely observed in human studies. This observation is probably due to the peculiarities of animal selection. The advantage of breeding polled goats generated a concerted effort of selection, with a systematic elimination of horned goats. This certainly generated a narrow bottleneck highly unusual in humans even in small populations. Such a situation makes it possible to better exploit LD and haplotype analysis to reach rapidly a gene-marker distance compatible with the search for coding sequences inside a BAC.

In a previous study (Vaiman et al. 1999b), it was shown that the 1-cM region encompassing PIS was homologous to the human region containing the Blepharophimosis Ptosis Epicanthus Syndrome (BPES) locus, a pathology combining premature ovarian failure (POF) and an excess of epiderm at the eyelids. The mapping data of our present paper do not contradict this homology. The recent mapping of a BPES-associated breakpoint inside a $45-\mathrm{kb}$ cosmid, and the relative position of the three genes $R B P 1, R B P 2$, and $C O P$, is completely consistent with the goat localization (De Baere et al. 1999). In this study the distance between RBP1 and COP is $\sim 100 \mathrm{~kb}$, whereas the distance between COP and the translocation can be summed up at $200 \mathrm{~kb}$. In our goat map, the distance between COP and CRBP is $<150 \mathrm{~kb}$, whereas the summation of $B A C$ fragments from COP to LSCV095 can be estimated at $<300 \mathrm{~kb}$. From these mapping data, we still cannot exclude the possibility that PIS and BPES are caused by the homologous gene. This is also corroborated by several physiological elements. In hornless goats, the two normal bony protuberances are apparent (Dove 1935), suggesting that only the epidermal counterpart fails to correctly induce the horn bud, which can be matched to the human palpebral anomaly in BPES. The POF ob- served in human BPES is somewhat more difficult to relate to the sexual reversion observed in PIS goats. Although sex reversion can involve late mechanisms, ovarian dysgenesis results more probably from early developmental mechanisms. The cloning of a gene having such a drastic effect on sex determination in some mammals (goats) and not in others (humans) will certainly shed new light on the sex determination pathway. We have started searching for coding sequences in the $376 \mathrm{H} 9 \mathrm{BAC}$ and hopefully will soon be able to give clear answers to this fascinating question.

\section{METHODS}

\section{BAC Library Screening, Restriction Analysis, and FISH Mapping of BAC Clones}

Clones were obtained from our goat BAC library using PCR and DNA prepared as described elsewhere (Schibler et al. 1998b). Insert size was evaluated by NotI digestion of $200 \mathrm{ng}$ of BAC DNA followed by field inversion gel electrophoresis (FIGE) on 1\% agarose gels. SalI restriction sites were identified likewise. FISH mapping experiments were carried out according to our classical protocol (Bahri-Darwich et al. 1994).

\section{Direct Sequencing of BAC Ends}

The Nucleobond AX100 kit (Macherey-Nagel) was used to prepare BAC DNA suitable for end sequencing, with slight modifications to the manufacturer's recommendations. Clones were grown for $16 \mathrm{hr}$ in $100 \mathrm{ml}$ of LB broth with $12.5 \mathrm{mg} / \mathrm{ml}$ chloramphenicol, starting from a single colony. BACs were midiprepped with $8 \mathrm{ml}$ of each buffer S1, S2, S3, and SDS was discarded using filters instead of centrifugation. The elution step was performed using $5 \times 1 \mathrm{ml}$ of $65^{\circ} \mathrm{C}$ preheated buffer N5. After isopropanol precipitation, DNA pellet was resuspended in $500 \mu \mathrm{l}$ of TE and then subjected to a second ethanol precipitation. Typical yield of $20 \mu \mathrm{g}$ was obtained with this protocol.

A total of $2.5 \mu \mathrm{g}$ of DNA template was added to $8 \mu \mathrm{l}$ of Big Dye Mix, 30 pmoles of T7L, or SP6L primers in an $11-\mu$ reaction volume. After a 3-min denaturation step at $95^{\circ} \mathrm{C}$, templates were subjected to 99 cycles of $30 \mathrm{sec}$ at $95^{\circ} \mathrm{C}, 20 \mathrm{sec}$ at $55^{\circ} \mathrm{C}$, and $4 \mathrm{~min}$ at $60^{\circ} \mathrm{C}$, using a Perkin-Elmer 9600 thermocycler. Samples were then loaded onto an ABI377 automated sequencer.

\section{BACs Subcloning and Microsatellite/STS Isolation}

About 500 ng of Nucleobond-prepared BAC were digested by Sau3A to completion and subcloned in a pGEM4z dephosphorylated vector. Sublibraries plated on $15-\mathrm{cm}$ Petri dishes were then screened with (TG) 12 and (TC) ${ }_{12}$ oligonucleotides labeled with the Boehringer DIG 3' end-labeling kit, according to our standard protocol (Schibler et al. 1998a). Sequencing of positive clones made it possible to design PCR primers, making it possible to amplify the microsatellite locus. Random clones were also picked and sequenced to develop PCR primers corresponding to STS.

\section{Family Structure, Genotyping, Recombinant Analysis, and LD Mapping}

Family structure was described in a previous paper (Vaiman et al. 1996a). Briefly, 12 polled bucks heterozygous for the mutation (Pp) were crossed with 211 horned females (pp) and 23 polled females ( $\mathrm{Pp}$ ) to generate 293 progeny (9-72 per buck). Females had one to three offspring (1.22 on average). These 
families were used previously to localize the PIS at goat chromosome $1 \mathrm{q} 43$. The males and the 234 females are not related and were therefore used to evaluate linkage disequilibrium for the closest PIS-flanking markers.

Radioactive genotyping was performed as described previously (Vaiman et al. 1996b). Marker order was determined both by the physical map and analysis of individual haplotypes. DISMULT and DISLAMB software (Terwilliger 1995) were used to estimate linkage disequilibrium parameters. For the DISMULT analysis, the parameters of the diseqin source file were 0.01 for the disease frequency, the distances between markers were fixed at 150, 20, 100, and $50 \mathrm{~kb}$ for LSCV082/ 095, LSCV095/098, LSCV098/LSCV202, and LSCV202/ LSCV203, respectively. LD was estimated at five points in each interval, and the maximal number of iterations was fixed at 800 .

\section{ACKNOWLEDGMENTS}

This work was funded by grants from INRA (AIP, sex autosomal determination in goats and pigs and AIP; Genome and Function). We acknowledge the constructive remarks formulated by both referees that permitted significant improvements of the manuscript.

The publication costs of this article were defrayed in part by payment of page charges. This article must therefore be hereby marked "advertisement" in accordance with 18 USC section 1734 solely to indicate this fact.

\section{REFERENCES}

Asdell, S.A. 1944. The genetic sex on intersexual goats and a probable linkage with the gene for hornlessness. Science 99: 124 .

Bahri-Darwich, I., D. Vaiman, I. Olsaker, A. Oustry, and E.P. Cribiu.

1994. Assignment of bovine synteny groups U27 and U8 to

R-banded chromosome 12 and 27, respectively. Hereditas 120: $261-265$.

Bourdelle, M.-E. 1903. Hermaphrodisme complexe des voies génitales et apparence masculine chez un bouc. Rev. Vét. Toulouse 11: $682-688$.

Chumakov, I.M., P. Rigault, I. Le Gall, C. Bellanne-Chantelot, A. Billault, S. Guillou, P. Soularue, G. Guasconi, E. Poullier, I. Gros et al. 1995. A YAC contig map of the human genome. Nature 377: 175-297.

Collignon, J., S. Sockanathan, A. Hacker, M. Cohen-Tannoudji, D. Norris, S. Rastan, M. Stevanovic, P.N. Goodfellow, and R. Lovell-Badge. 1996. A comparison of the properties of Sox-3 with Sry and two related genes, Sox-1 and Sox-2. Development 122: 509-520.

De Baere, E., N. Van Roy, F. Speleman, Y. Fukushima, A. De Paepe, and L. Messiaen. 1999. Closing in on the BPES gene on 3q23: Mapping of a de novo reciprocal translocation $\mathrm{t}(3 ; 4)(\mathrm{q} 23 ; \mathrm{p} 15.2)$ breakpoint within a $45-\mathrm{kb}$ cosmid and mapping of three candidate genes, RBP1, RBP2, and beta'-COP, distal to the breakpoint. Genomics 57: 70-78.

de la Chapelle, A. and F.A. Wright. 1998. Linkage disequilibrium mapping in isolated populations: The example of Finland revisited. Proc. Natl. Acad. Sci. 95: 12416-12423.

Deng, H.X., K. Abe, I. Kondo, M. Tsukahara, H. Inagaki, I. Hamada, Y. Fukushima, and N. Niikawa. 1991. Parental origin and mechanism of formation of polysomy X: An XXXXX case and four XXXXY cases determined with RFLPs. Hum. Genet. 86: $541-544$.

Dove, W.L. 1935. Physiology of horn growth. J. Exp. Zool. 69: 347-404.

Dunner, S., C. Charlier, F. Farnir, B. Brouwers, J. Canon, and M. Georges. 1997. Towards interbreed IBD fine mapping of the $\mathrm{mh}$ locus: Double-muscling in the Asturiana de los Valles breed involves the same locus as in the Belgian Blue cattle breed. Mamm. Genome 8: 430-435.

Foster, J.W., M.A. Dominguez-Steglich, S. Guioli, G. Kowk, P.A.
Weller, M. Stevanovic, J. Weissenbach, S. Mansour, I.D. Young, P.N. Goodfellow et al. 1994. Campomelic dysplasia and autosomal sex reversal caused by mutations in an SRY-related gene. Nature 372: 525-530.

Hudson, T.J., L.D. Stein, S.S. Gerety, J. Ma, A.B. Castle, J. Silva, D.K. Slonim, R. Baystista, L. Krughjaku, and S.H. Xu. 1995. An STS-based map of the human genome Science 270: 1945-1954.

Koopman, P., J. Gubbay, N. Vivian, P. Goodfellow, and R. Lovell-Badge. 1991. Male development of chromosomally female mice transgenic for Sry. Nature 351: 117-121.

Petit, G. 1894. Nouvelle observation d'hermaphrodisme complexe des voies génitales chez un bouc. Recueil de Médecine Vétérinaire, Ecole d'Alfort 71: 247-249.

Ricordeau, G. and J.-J. Lauvergne. 1967. Hypothèse génétique unique pour expliquer la présence d'intersexués, de màles en excès et de mâles stériles en race caprine saanen. Ann. Zootechnie 16: 323-334.

Riquet, J., W. Coppieters, N. Cambisano, J.J. Arranz, P. Berzi, S.K. Davis, B. Grisart, F. Farnir, L. Karim, M. Mni et al. 1999. Fine-mapping of quantitative trait loci by identity by descent in outbred populations: Application to milk production in dairy cattle. Proc. Natl. Acad. Sci. 96: 9252-9257.

Schibler, L., D. Vaiman, A. Oustry, C. Giraud-Delville, and E.P. Cribiu. 1998a. Comparative gene mapping: A fine-scale survey of chromosome rearrangements between ruminants and humans. Genome Res. 8: 901-915.

Schibler, L., D. Vaiman, A. Oustry, N. Guinec, A.L. Dangy-Caye, A. Billault, and E.P. Cribiu. 1998b. Construction and extensive characterization of a goat bacterial artificial chromosome library with threefold genome coverage. Mamm. Genome 9: 119-124.

Soller, M., B. Padeh, M. Wysoki, and N. Ayalon. 1969. Cytogenetics of Saanen goats showing abnormal development of the reproductive tract associated with the dominant gene for polledness. Cytogenetics 8: 51-67.

Stevanovic, M., R. Lovell-Badge, J. Collignon, and P.N. Goodfellow. 1993. SOX3 is an X-linked gene related to SRY. Hum. Mol. Genet. 2: 2013-2018.

Swain, A. and R. Lovell-Badge. 1999. Mammalian sex determination: A molecular drama. Genes \& Dev. 13: 755-767.

Terwilliger, J.D. 1995. A powerful likelihood method for the analysis of linkage disequilibrium between trait loci and one or more polymorphic marker loci. Am. J. Hum. Genet. 56: 777-787.

Tommerup, N., W. Shhempp, P. Meinecke, S. Pedersen, L. Bolund et al. 1993. Assignment of an autosomal sex reversal locus (SRA1) and campomelic dysplasia (CMPD1) to 17q24.3-q25.1. Nat. Genet. 4: 170-173.

Vaiman, D., O. Koutita, A. Oustry, J.M. Elsen, E. Manfredi, M. Fellous, and E.P. Cribiu. 1996a. Genetic mapping of the autosomal region involved in XX sex-reversal and horn development in goats. Mamm. Genome 7: 133-137.

Vaiman, D., L. Schibler, F. Bourgeois, A. Oustry, Y. Amigues, and E.P. Cribiu. 1996b. A genetic linkage map of the male goat genome. Genetics 144: 279-305.

Vaiman, D., A. Billault, K. Tabet-Aoul, L. Schibler, D. Vilette, C. Soravito, and E.P. Cribiu. 1999a Construction and characterization of a sheep BAC library of three genome equivalents. Mamm. Genome 10: 585-587.

Vaiman, D., L. Schibler, A. Oustry-Vaiman, E. Pailhoux, T. Goldammer, M. Stevanovic, J.P. Furet, M. Schwerin, C. Cotinot, M. Fellous et al. 1999b. High-resolution Human/Goat comparative map of the goat Polled/Intersex syndrome (PIS): The human homologue is contained in a human YAC from HSA3q23. Genomics 56: 31-39.

Zanaria, E., B. Bardoni, B. Dabovic, V. Calvari, M. Fraccaro, O. Zuffardi, and G. Camerino. 1995. Xp duplications and sex reversal. Philos. Trans. R. Soc. Lond. B. Biol. Sci. 350: 291-296.

Zlotogora, J., M. Sagi, and T. Cohen. 1983. The blepharophimosis, ptosis, and epicanthus inversus syndrome: Delineation of two types. Am. J. Hum. Genet. 35: 1020-1027.

Received September 28, 1999; accepted in revised form January 20, 2000.

\section{Genome Research}




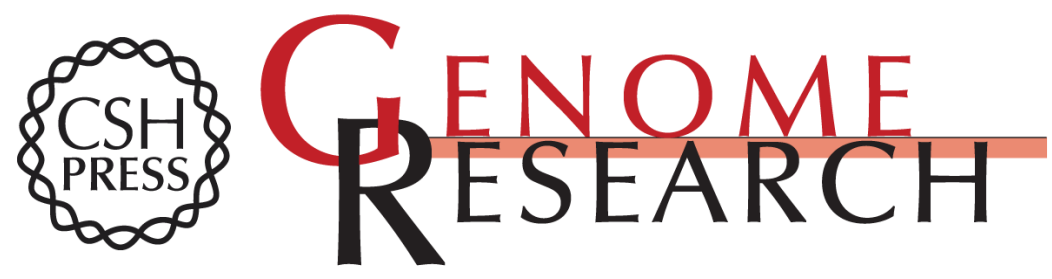

\section{Fine Mapping Suggests that the Goat Polled Intersex Syndrome and the Human Blepharophimosis Ptosis Epicanthus Syndrome Map to a 100-kb Homologous Region}

Laurent Schibler, Edmond P. Cribiu, Anne Oustry-Vaiman, et al.

Genome Res. 2000 10: 311-318

Access the most recent version at doi:10.1101/gr.10.3.311

References This article cites 26 articles, 8 of which can be accessed free at: http://genome.cshlp.org/content/10/3/311.full.html\#ref-list-1

\section{License}

Email Alerting

Receive free email alerts when new articles cite this article - sign up in the box at the Service top right corner of the article or click here.

\section{Affordable, Accurate Sequencing.}

